\title{
Synthesis and Mechanistic Study of Palladium Nanobars and Nanorods
}

\author{
Yujie Xiong, ${ }^{\dagger}$ Honggang Cai, ${ }^{\dagger}$ Benjamin J. Wiley, ${ }^{\dagger}$ Jinguo Wang, ${ }^{\S}$ \\ Moon J. Kim, ${ }^{\S}$ and Younan Xia ${ }^{\dagger} *$
}

\footnotetext{
${ }^{\dagger}$ Department of Chemistry, University of Washington, Seattle, Washington 98195

${ }^{*}$ Department of Chemical Engineering, University of Washington, Seattle, Washington 98195

${ }^{\S}$ Department of Electrical Engineering, University of Texas at Dallas, Richardson, Texas 75083

*Corresponding author. E-mail: xia@chem.washington.edu
} 

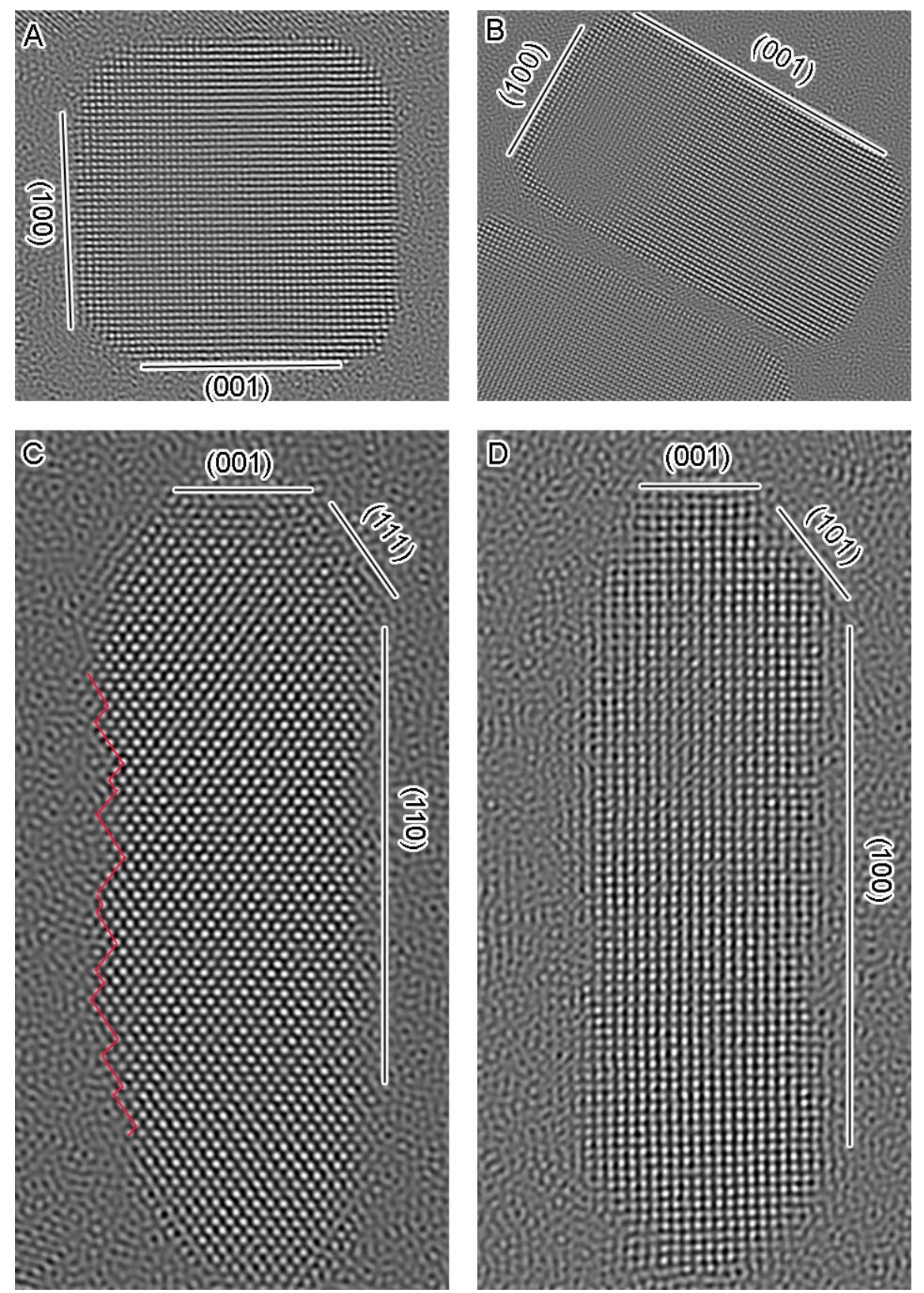

Figure S1. High-resolution TEM images that were obtained by filtering the images in Figure 2 with a Gatan Digital Micrograph program: (A) a nanobar with an aspect ratio of $\sim 1$ recorded along [010] axis; (B) a nanobar with an aspect ratio of $\sim 2$ recorded along [010] axis; (C) a nanorod recorded along [110] axis; and (D) a nanorod recorded along [010] axis. The red line in image-C indicates a rough surface resulting from the reconstruction of $\{110\}$ to $\{111\}$. 


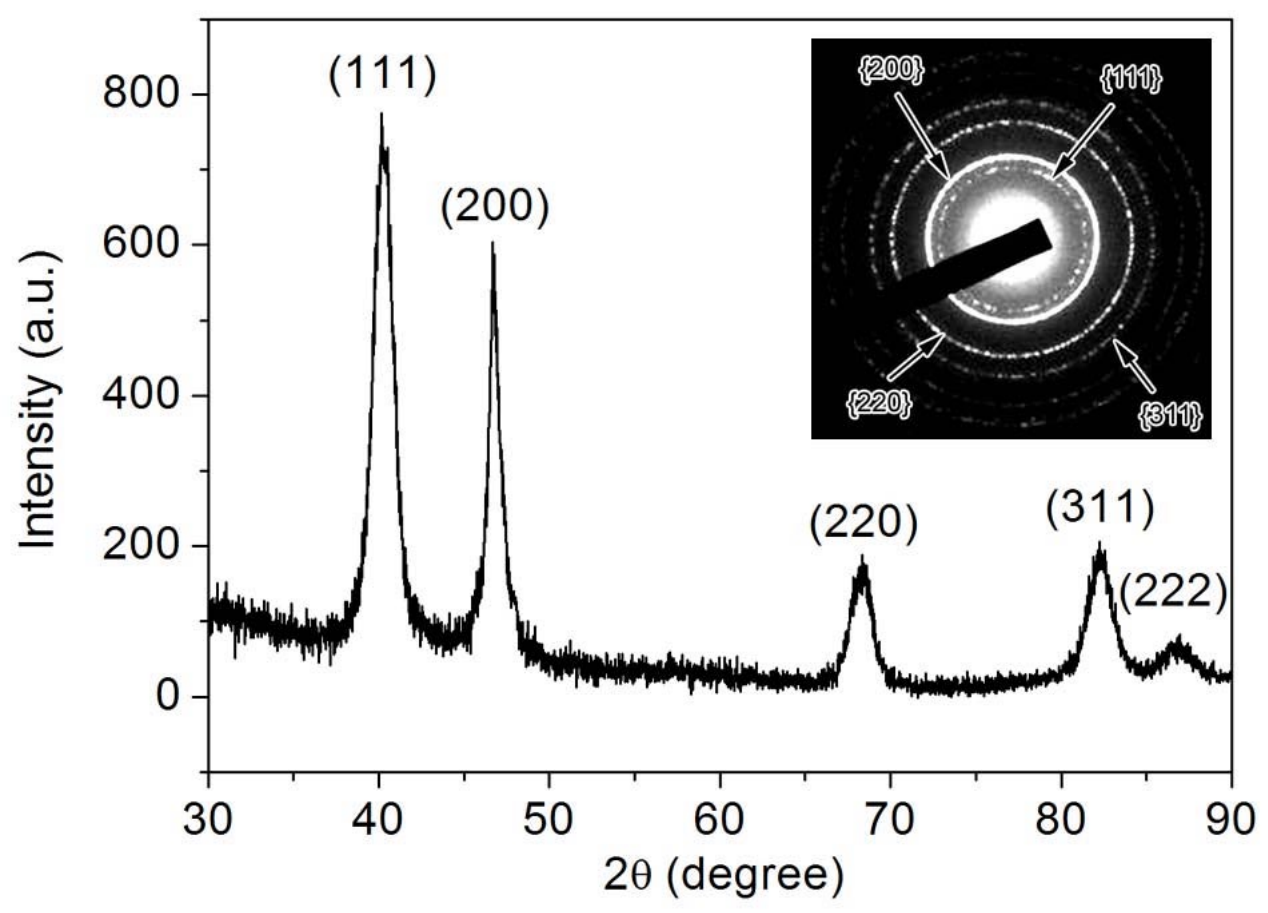

Figure S2. XRD pattern taken from Pd nanobars shown in Figure 1B. All the peaks can be indexed to $f c c$ palladium (JCPDS card, 05-0681). No peaks were observed for impurities such as $\mathrm{PdBr}_{2}$ and $\mathrm{PdO}$. The ratio between the intensities of (111) and (200) peaks is much lower than the value reported for a conventional powder sample (1.33 versus 2.38), indicating that the diffraction from $\{100\}$ planes is enhanced for the sample of nanobars. It is likely that the nanobars preferentially lay on the substrate against their flat $\{100\}$ facets. As a result, there was a texturing effect. The ED pattern (inset) taken from an assembly of Pd nanobars also indicate that they were highly crystalline. The rings can be indexed to the diffraction from $\{111\},\{200\}$, $\{220\}$ and $\{311\}$ planes of $f c c$ palladium, respectively. 

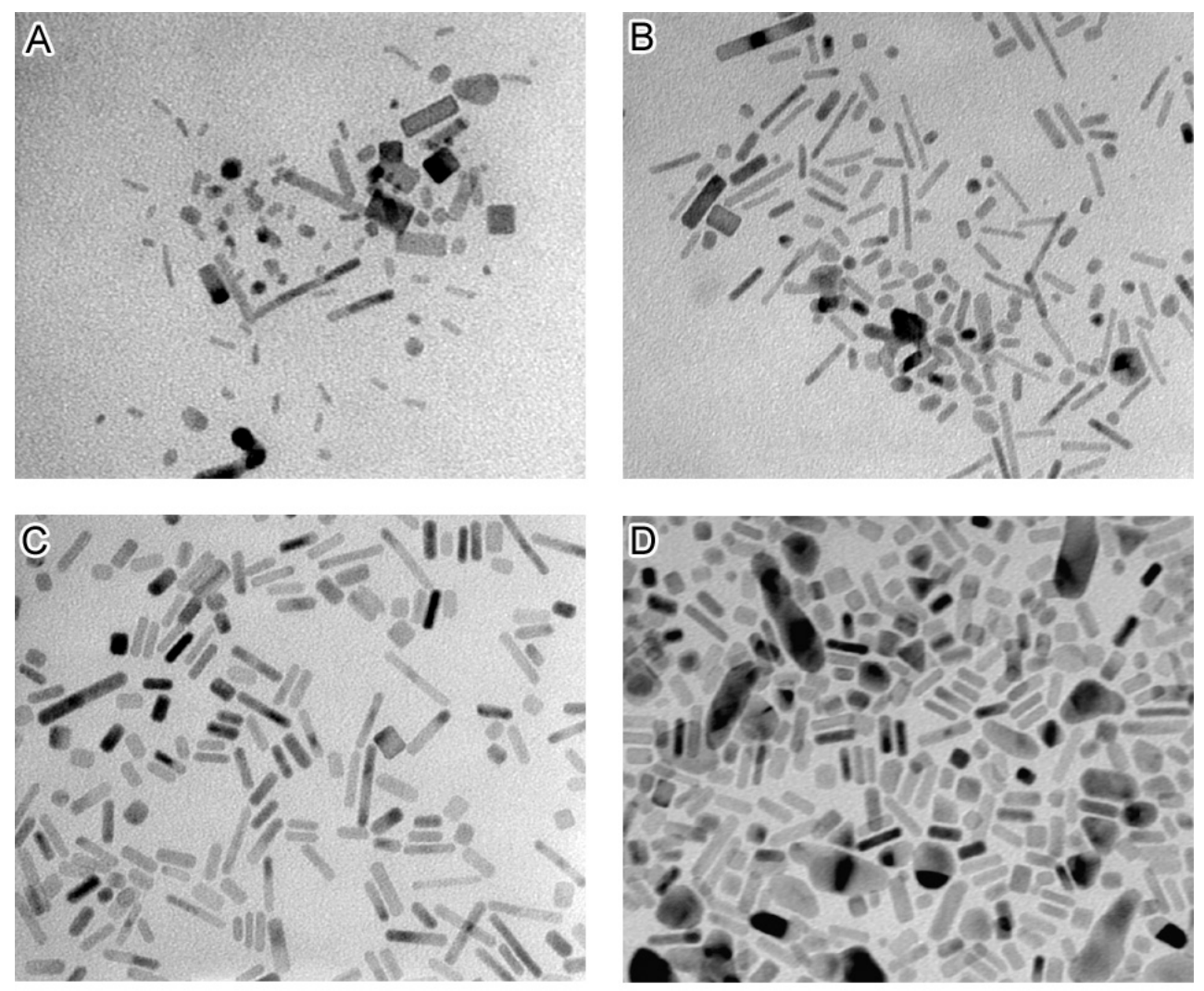

$20 \mathrm{~nm}$

Figure S3. TEM images of $\mathrm{Pd}$ nanoparticles obtained with precursor $\mathrm{Na}_{2} \mathrm{PdBr}_{4}$ in the presence of $\mathrm{KBr}$ at different concentrations. The molar ratio of $\mathrm{KBr}$ to $\mathrm{Na}_{2} \mathrm{PdBr}_{4}$ was: (A) 8 ; (B) 15 ; (C) 30; and (D) 50. All the syntheses were conducted with $72.7 \%$ EG in an 11-mL mixture of EG and water at $100{ }^{\circ} \mathrm{C}$, and in the presence of $75 \mathrm{mM}$ PVP. These parameters were the same as those in Figure 4, except the use of $\mathrm{Na}_{2} \mathrm{PdBr}_{4}$ instead of $\mathrm{Na}_{2} \mathrm{PdCl}_{4}$ as a precursor compound. The morphology exhibited a similar dependence on the concentration of $\mathrm{KBr}$. As compared to the case with $\mathrm{Na}_{2} \mathrm{PdCl}_{4}$ as a precursor, the samples prepared with $\mathrm{Na}_{2} \mathrm{PdBr}_{4}$ had lower aspect ratios. This difference can be attributed to the fact that oxidative etching became weaker in the absence of chloride, and thus it is more difficult to activate the surface of nanocrystals. 


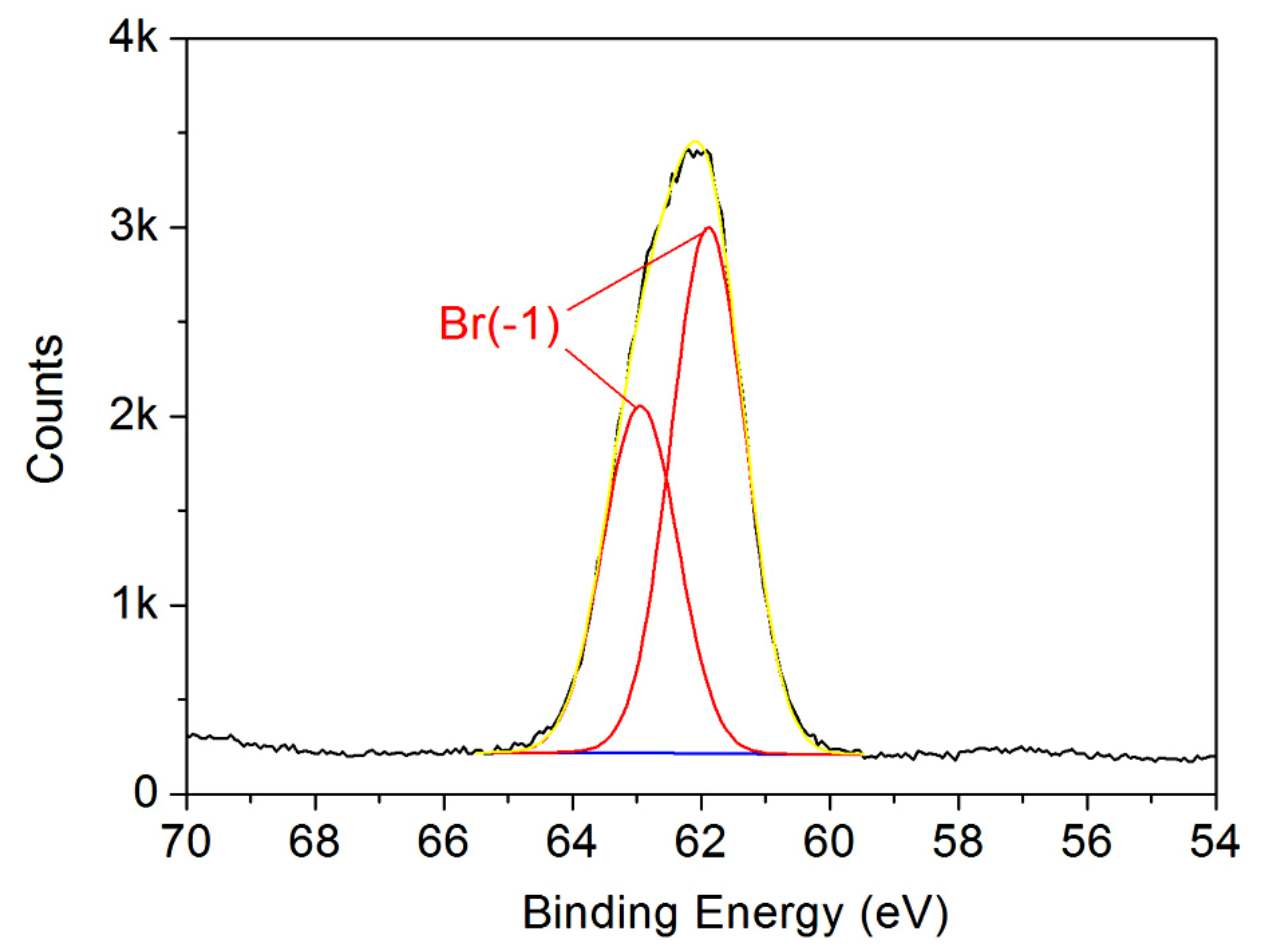

Figure S4. High-resolution XPS core level spectrum for the $\mathrm{Br}(3 \mathrm{~d})$ in $\mathrm{KBr}$. 

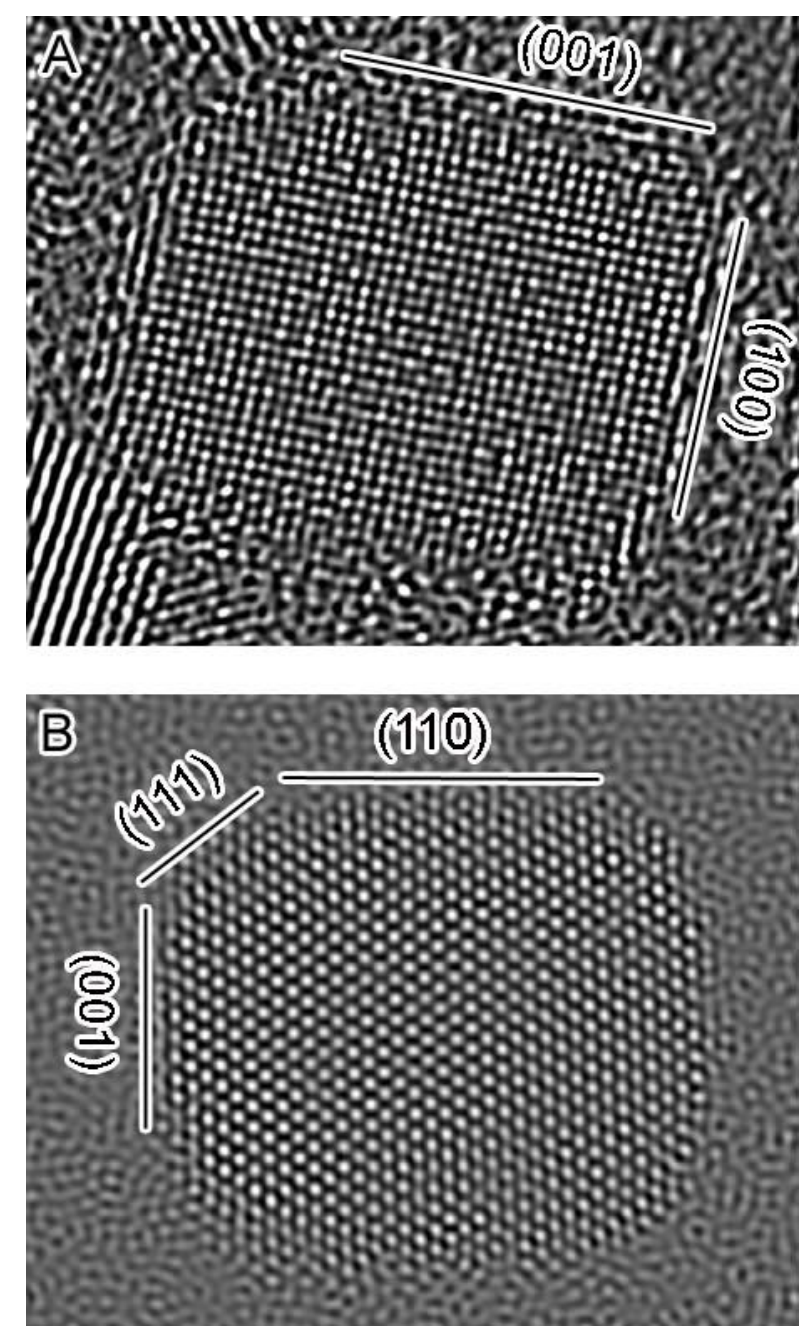

Figure S5. High-resolution TEM images that were obtained by filtering the images in Figure 8 with a Gatan Digital Micrograph program: (A) a Pt nanobar recorded along [010] axis; and (B) a Pt nanobar recorded along [11̄0] axis. 


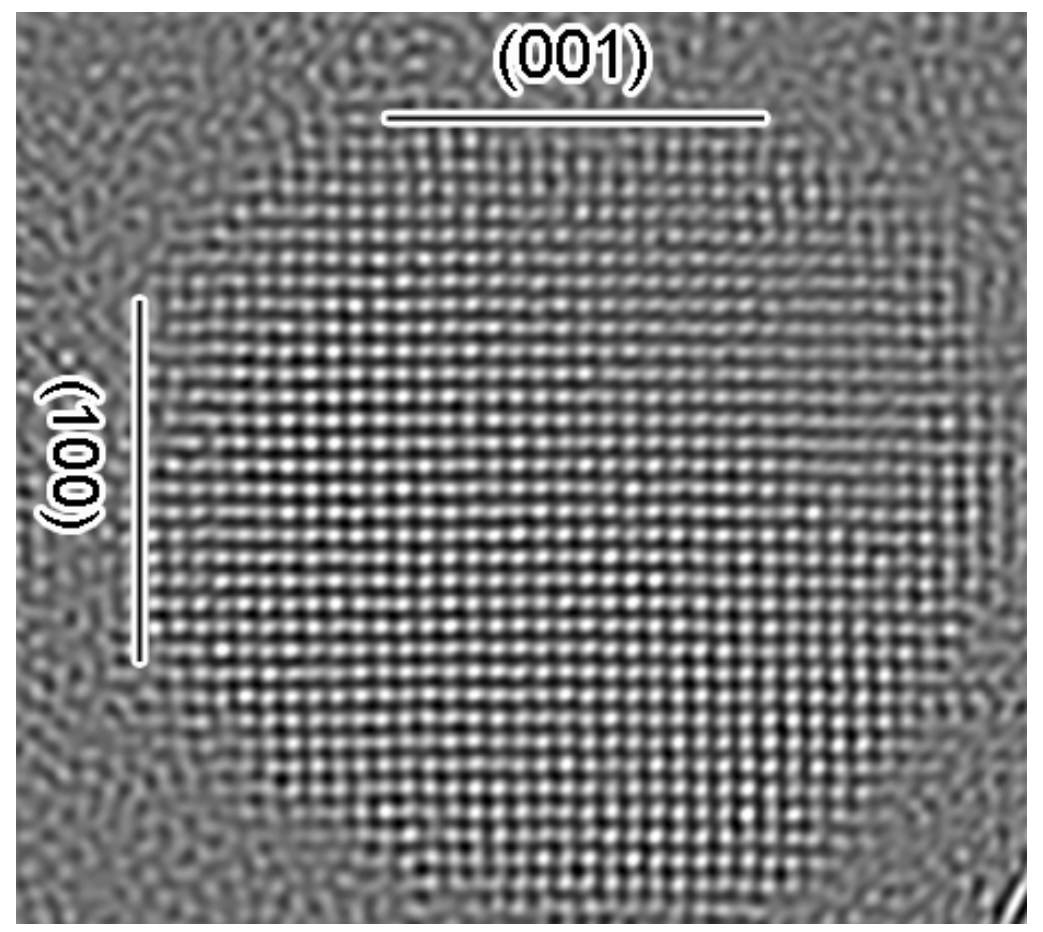

Figure S6. High-resolution TEM image of a cubooctahedron formed through an aging process. This image was obtained by filtering the image in Figure 9D with a Gatan Digital Micrograph program. 

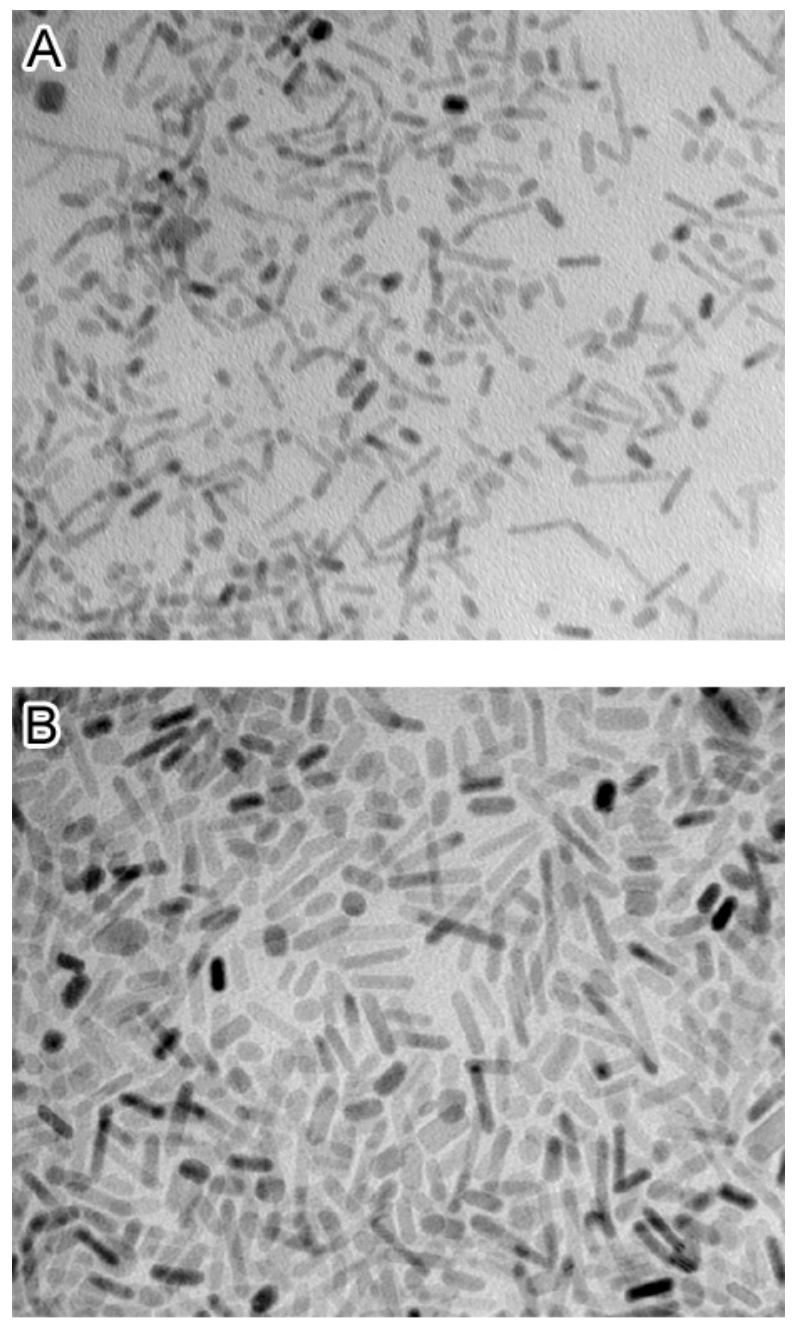

$-20 \mathrm{~nm}$

Figure S7. TEM images of samples prepared under the same condition as in Figure $1 \mathrm{D}(\mathrm{t}=1 \mathrm{~h})$ except that the reaction time was (A) shortened to $10 \mathrm{~min}$; and (B) prolonged to $3 \mathrm{~h}$, respectively. At $\mathrm{t}=10 \mathrm{~min}$, the sample mainly contained nanorods $(1.8 \mathrm{~nm} \times 12 \mathrm{~nm})$, suggesting that the nanorods shown in Figure 1 were formed through nucleation and growth (as depicted in Figure 7B) rather than evolution from nanobars during an aging process (Figure 7C). During the next $50 \mathrm{~min}$, the nanorods slowly grew to $2 \mathrm{~nm} \times 16 \mathrm{~nm}$. If the reaction was allowed to proceed to $\mathrm{t}=$ $3 \mathrm{~h}$, the diameter of nanorods increased while their length decreased, indicating that an aging process occurred between $\mathrm{t}=1 \mathrm{~h}$ and $\mathrm{t}=3 \mathrm{~h}$. 